\title{
ВЛИЯНИЕ ТРАНСТУМОРАЛЬНОЙ ДЕКОМПРЕССИИ НА ЦЕНТРАЛЬНУЮ ГЕМОДИНАМИКУ И ВНУТРИБРЮШНОЕ ДАВЛЕНИЕ У БОЛЬНЫХ С ОСТРОЙ ОБТУРАЦИОННОЙ ТОЛСТОКИШЕЧНОЙ НЕПРОХОДИМОСТЬЮ
}

\section{CENTRAL HEMODYNAMICS AND INTRA- ABDOMINAL PRESSURE IN PATIENTS WITH ACUTE OBSTRUCTIVE COLONIC OBSTRUCTION}

\section{Bagdasarov}

E. Bagdasarova

A. Eleev

Summary. The article presents the results of a study of central hemodynamics in 32 patients with acute colonic obstruction (OTKN) of tumor origin. In 28 patients, OTKN was resolved by endoscopic decompression-transtumoral stenting (self-expanding metallic stents (SEMS)). Central hemodynamic studies have shown that endoscopic decompression (transtumoral stenting) with OTKN, it allows all patients with compensated and subcompensated $0 T K N$ to restore hemodynamic parameters within 5-7 days, and in the most severe patients with decompensated intestinal obstruction, to bring the normalization period closer only to $10-12$ days after decompression.

Keywords: acute colonic obstruction, intra-abdominal pressure, intraabdominal hypertension, transtumoral stenting.

\author{
Багдасаров Валерий Вартанович \\ Д.м.н., профессор, Российский Университет Дружбы \\ Народов \\ Багдасарова Елена Анатольевна \\ Д.м.н., заведующая отделением, ДЗМ ГКБ С.С. Юдина
}

Елеев Алим Анатольевич

Врач хирург, колопроктолог, онколог, ДЗМ ГКБ

С.С. Юдина

Alimeleev@gmail.com

Аннотация. В статье приведены результаты исследования центральной гемодинамики у 32 больных с острой толстокишечной непроходимостью (ОТКН) опухолевого генеза. У 28 больных ОТКН была разрешена эндоскопической декомпрессией-транстуморальным стентированием (саморасширяющиеся сетчатые стенты (self-expanding metallic stents SEMS). Исследования центральной гемодинамики показали, что эндоскопическая декомпрессия (транстуморальное стентирование) при ОТКН позволяет у всех пациентов с компенсированной и субкомпенсированной ОТКН восстановить гемодинамические показатели в течение 5-7 суток, а у наиболее тяжелых больных сдекомпенсированной кишечной непроходимостью приблизить сроки нормализации только к 10-12 суткам после декомпрессии.

Ключевые слова: острая толстокишечная непроходимость, внутрибрюшное давление, интраабдоминальная гипертензия, транстуморальное стентирование.
B нутрибрюшное давление (ВБД) как фактор гомеостаза привлекает внимание исследователей более ста лет [13, с. 509; 14, с. 989].

Одним из основных этиологических факторов повышения ВБД является кишечная непроходимость [10, C. 572].

Многочисленные исследования на животных и человеке показали отрицательное влияние повышенного ВБД на функцию сердечно-сосудистой системы. Рост компрессии в брюшной полости замедляет кровоток по нижней полой вене и уменьшает венозный возврат, ограничивает диастолическое заполнение желудочков, способствует повышению ЦВД. При дальнейшем прогрессировании интраабдоминальной гипертензии отмечалось снижение ударного объема и сердечного выброса (CB), несмотря на компенсаторную тахикардию. Сокращение органного кровотока не пропорционально уменьшению СВ и развивается раньше [3, с. 147;4, с. $938 ; 9$, с. $43 ; 10$, с. $553 ; 15$, с. 917$]$.

Увеличение ОПСС было прямо пропорционально величине интраабдоминальной гипертензии (ИАГ) и связано с прямым механическим воздействием на сосуды брюшной полости. Среднее артериальное давление у больных ИАГ снижалось по мере увеличения ВБД $[17$, с. $31 ; 6$, с. $1354 ; 16$, с. $64 ; 14$, с. 989]. 
Таблица 1. Данные об основных параметрах центральной гемодинамики у больных с ОТКН

\begin{tabular}{|c|c|c|c|c|}
\hline \multirow{2}{*}{ Показатель } & \multirow{2}{*}{ Контроль } & \multicolumn{3}{|c|}{ Степень динамической кишечной непроходимости } \\
\hline & & Компенс. & Субкомпенс. & Декомпенс. \\
\hline 1.КДР, мм & $\begin{array}{l}52,2 \pm 0,6 \\
p>0,05\end{array}$ & $\begin{array}{l}52,6 \pm 0,6 \\
p>0,05 \\
p_{1}>0,05\end{array}$ & $\begin{array}{l}51,0 \pm 0,4 \\
p>0,05 p_{1}<0,05\end{array}$ & $\begin{array}{l}51,2 \pm 0,6 \\
p>0,05 \\
p_{1}<0,05 \\
p_{2}>0,05 \\
\end{array}$ \\
\hline 2.KCP, MM & $32,2 \pm 0,8$ & $\begin{array}{l}34,6 \pm 0,4 \\
p>0,05\end{array}$ & $\begin{array}{l}36,6 \pm 0,6 \\
p<0,01 p_{1}<0,01\end{array}$ & $\begin{array}{l}39,4 \pm 0,4 \\
\mathrm{p}<0,01 \\
\mathrm{p}_{1}<0,01 \\
\mathrm{p}_{2}<0,01\end{array}$ \\
\hline 3.КДО, $\mathrm{cm}^{3}$ & $128,8 \pm 3,8$ & $\begin{array}{l}134,2 \pm 2,2 \\
p<0,05\end{array}$ & $\begin{array}{l}124,2 \pm 2,8 \\
\mathrm{p}>0,05 \mathrm{p}_{1}<0,05\end{array}$ & $\begin{array}{l}125,6 \pm 3,1 \\
p>0,05 p_{1}<0,05 \\
p_{2}>0,05\end{array}$ \\
\hline 4.KCO, $\mathrm{CM}^{3}$ & $45,2 \pm 2,0$ & $\begin{array}{l}48,2 \pm 2,0 \\
p>0,05\end{array}$ & $\begin{array}{l}58,2 \pm 1,2 \\
\mathrm{p}<0,01 \mathrm{p}_{1}<0,01\end{array}$ & $\begin{array}{l}67,8 \pm 1,8 \\
\mathrm{p}<0,01 \mathrm{p}_{1}<0,01 \\
\mathrm{p}_{2}<0,01\end{array}$ \\
\hline 5.УО, мл & $84,4 \pm 2,8$ & $\begin{array}{l}84,6 \pm 2,6 \\
p>0,05\end{array}$ & $\begin{array}{l}64,6 \pm 3,4 \\
p<0,01 p_{1}<0,01\end{array}$ & $\begin{array}{l}58,6 \pm 2,4 \\
p<0,01 p_{1}<0,01 \\
p_{2}>0,05\end{array}$ \\
\hline $6 . Ф \mathrm{~B}, \%$ & $64,2 \pm 1,8$ & $\begin{array}{l}64,2 \pm 0,8 \\
p>0,05\end{array}$ & $\begin{array}{l}54,6 \pm 2,2 \\
p<0,01 p_{1}<0,01\end{array}$ & $\begin{array}{l}45,8 \pm 2,4 \\
\mathrm{p}<0,01 \mathrm{p}_{1}<0,01 \\
\mathrm{p}_{2}>0,05\end{array}$ \\
\hline 7.\%FS & $36,2 \pm 0,2$ & $\begin{array}{l}35,0 \pm 0,4 \\
p>0,05\end{array}$ & $\begin{array}{l}27,8 \pm 2,2 \\
p<0,01 p_{1}<0,01\end{array}$ & $\begin{array}{l}24,8 \pm 1,4 \\
\mathrm{p}<0,01 \mathrm{p}_{1}<0,01 \\
\mathrm{p}_{2}>0,05\end{array}$ \\
\hline 8.MOC, л & $5,4 \pm 0,4$ & $\begin{array}{l}7,6 \pm 0,2 \\
p<0,01\end{array}$ & $\begin{array}{l}6,2 \pm 0,2 \\
\mathrm{p}>0,05 \mathrm{p}_{1}<0,01\end{array}$ & $\begin{array}{l}5,6 \pm 0,2 \\
\mathrm{p}>0,05 \\
\mathrm{p}_{1}<0,01\end{array}$ \\
\hline $9.4 С С$, уд/мин & $62,8 \pm 2,8$ & $\begin{array}{l}86,6 \pm 1,8 \\
p<0,01\end{array}$ & $\begin{array}{l}92,4 \pm 2,2 \\
\mathrm{p}<0,01 \mathrm{p}_{1}<0,01\end{array}$ & $\begin{array}{l}99,8 \pm 2,2 \\
p<0,01 p_{1}<0,01 \\
p_{2}<0,01\end{array}$ \\
\hline 10.АД/ср, мм рт. ст. & $97,4 \pm 1,2$ & $\begin{array}{l}97,0 \pm 1,6 \\
p>0,05\end{array}$ & $\begin{array}{l}96,8 \pm 1,2 \\
p>0,05 p_{1}>0,01\end{array}$ & $\begin{array}{l}96,8 \pm 1,3 \\
p>0,05 \\
p_{1}>0,01 p_{2}>0,05\end{array}$ \\
\hline $\begin{array}{l}\text { 11.СИ } \\
\text { л/мин } \times \mathrm{M}^{2}\end{array}$ & $3,2 \pm 0,1$ & $\begin{array}{l}4,2 \pm 0,8 \\
p<0,05\end{array}$ & $\begin{array}{l}3,4 \pm 0,2 \\
\mathrm{p}>0,05 \mathrm{p}_{1}<0,05\end{array}$ & $\begin{array}{l}3,2 \pm 0,4 \\
\mathrm{p}>0,05 \\
\mathrm{p}_{1}<0,05 \\
\mathrm{p}_{2}>0,05\end{array}$ \\
\hline $\begin{array}{l}\text { ОПСС } \\
\text { дин с.сек-5 }\end{array}$ & $1426,4 \pm 44,5$ & $\begin{array}{l}1038,3 \pm 38,5 \\
p<0,01\end{array}$ & $\begin{array}{l}1276,2 \pm 90,8 \\
p>0,05 p_{1}<0,05\end{array}$ & $\begin{array}{l}1422,4 \pm 46,8 \\
\mathrm{p}>0,05 \mathrm{p}_{1}<0,01 \mathrm{p}_{2}>0,05\end{array}$ \\
\hline
\end{tabular}

Развитие гиповолемии, артериальной гипотензии, гипоксемии может вести к снижению абдоминально-перфузионного давления (АПД) и гипоперфузии кишечника, что является триггером развития дисфункции желудочно-кишечного тракта $[18$, с. $101 ; 8$, с. $14 ; 16$, с. $77 ; 15$, с. $918 ; 9$, с. 53$]$.

Внедрение эндоскопических технологий, интегральных шкал с объективной оценкой тяжести состояния больных и прогноза исхода привели к значительным изменениям в этапном хирургическом лечения острой толстокишечной непроходимости (ОТКН) с приорите- том первичной декомпрессии ободочной кишки (транстуморальное стентирование саморасправлющимися металлическими стентами -Self-expandable metal stent SEMS), позволяющая существенно снизить пооперационную летальность [7, с. 475].

\section{Цe^ь}

Изучить нарушения центральной гемодинамики и мониторинг внутрибрюшного давления у больных с ОТКН до и после транстуморальной декомпрессии (стентирование кишки). 


\section{Материал и методы}

исслеАования

Материалом настоящей работы являются результаты лечения 32 больных с ОТКН в период с 2016-2018 г. в ГКБ им. С.С. Юдина, у которых на первом этапе хирургического лечения была выполнена эндоскопическая декомпрессия (транстуморальное стентирование ободочной кишки). У больных этой группы были использованы саморасширяющиеся сетчатые стенты (selfexpanding metallic stents - SEMS). Средний возраст составил 58,4 $\pm 9,6$ лет.

Нарушение центральной гемодинамики и внутрибрюшное давление (ВБД) исследовали у 32 больных с ОТКН до и после транстуморальной декомпрессии. В первую группу включили 8 пациентов с компенсированной ОТКН; во вторую вошли 16 больных с субкомпенсированной ОТКН, и 8 больных с декомпенсированной ОТКН составили 3 группу.

Больным с различной степенью ОТКН после эндоскопической декомпрессии с целью изучения восстановления показателей центральной гемодинамики и определения сроков радикального оперативного лечения провели исследование гемодинамических параметров на 3, 7, 10 сутки после выполнения декомпрессии кишки.

Для исследования работы сердечно-сосудистой системы использовали клинические данные и данные эхокардиографии (ЭхоКГ).

Эхо КГ выполняли на аппарате «LOGIQ 400MD фирмы «General Electric» (США), а ЭКГ на 6-канальном электрокардиографе «Delchi».

\section{Результаты и обсужАение}

Тяжесть нарушений гемодинамики зависела от степени тяжести состояния (APACHEII) и степени ОТКН.

В первой группе с компенсированной ОТКН у 8 больных было 10 баллов по АРАCHE II, во второй, с субкомпенсированной ОТКН у 16 больных от 11-20 баллов и в третьей у 8 больных с декомпенсированной степенью ОТКН-от 21-30 баллов АРАСНЕ II.

До эндоскопической транстуморальной декомпрессии в первой группе ВБД было - 12,8 $\pm 1,8$ мм рт. ст., во второй группе показатели ВБД $=16,2 \pm 3,2$ мм рт. ст. и в третьей группе ВБД =19,3 44,2 мм рт. ст.

После эндоскопической декомпрессии в первой группе ВБД снизилось до $-8,2 \pm 0,8$ мм рт. ст., во вто- рой группе ВБД $=11,2 \pm 2,82$ мм рт. ст.; в третьей группе $=12,4 \pm 2,42$ мм рт.ст.

Данные об основных параметрах центральной гемодинамики у больных с ОТКН до транстуморальной декомпрессии представлены в таблице № 1.

Снижение сократительной активности миокарда проявлялось увеличением конечно-систолического размера (КСР) левого желудочка до 36,6士 0,6 $(p<0,01)$ в группе больных с субкомпенсированной, и до $39,4 \pm 0,4(p<0,01)$ - декомпенсированной ОТКН.

В результате увеличения КСР у больных обнаруживали увеличение конечно-систолического объема (KCO).

Различия с показателями: $\mathrm{p}-\mathrm{c}$ контрольной группой; р1- с компенсированной ДКН; р2- с субкомпенсированной

Так, если увеличение КСО до 48,2+2,0 (p>0,05) при компенсированной

ОТКН не было достоверно значимым, то в группах с субкомпенсированной и декомпенсированной ОТКН выявили значительное увеличение КСО (p $<0,01)$ до $58,2 \pm 1,2$ см3 ( $p<0,01)$ и до $67,8 \pm 1,8$ см3 соответственно(табл.1).

Снижение фракции выброса (ФВ) и степень укорочения передне-заднего размера полости левого желудочка во время систолы (\%FS) отражали нарушения сократительной активности миокарда, что было характерно для суб- и декомпенсированной ОТКН. При субкомпенсированной ОТКН обнаружили снижение ФВ до $54,6 \pm 2,2 \%(p<0,01)$, при декомпенсиро-

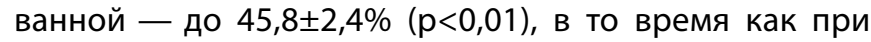
компенсированной ОТКН снижение ФВ не имело достоверных отличий от контрольной группы.\% FS была достоверно снижена при суб- и декомпенсированной ОТКН $-26,8 \pm 2,2 \%(p<0,01)$ и $24,8 \pm 1,8 \%(p<0,01)$, соответственно.

Если у больных с суб- и декомпенсированной ОТКН при увеличении ЧСС регистрировали снижения удар-

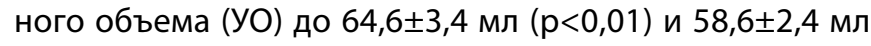
$(p<0,01)$, соответственно, то у больных с компенсиро-

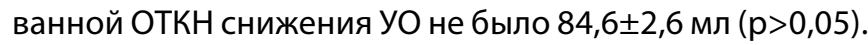

Уменьшение УО и тахикардия у большинства больных с компенсированной и субкомпенсированной ОТКН обнаружили гипердинамический тип кровообращения с увеличением минутного объема (МОC)

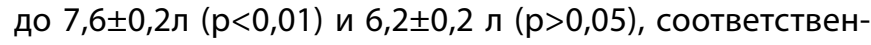


Таблица 2. Данные об изменении центральной гемодинамики у больных компенсированной ОТКН после декомпрессии кишки

\begin{tabular}{|c|c|c|c|}
\hline Показатель & Контроль & $\begin{array}{l}\text { До стентирования } \\
\text { кишки }\end{array}$ & $\begin{array}{l}3 \text { сутки } \\
\text { после стентирования }\end{array}$ \\
\hline КДР, мм & $\begin{array}{l}52,2 \pm 0,6 \\
p>0,05\end{array}$ & $\begin{array}{l}52,6 \pm 0,6 \\
p>0,05\end{array}$ & $\begin{array}{l}52,4 \pm 0,6 \\
p>0,05 p_{1}>0,05\end{array}$ \\
\hline KCP, MM & $32,2 \pm 0,8$ & $34,8 \pm 0,2 p>0,05$ & $34,5 \pm 0,6 p>0,05 p_{1}>0,05$ \\
\hline КДО, $\mathrm{cm}^{3}$ & $128,8 \pm 3,8$ & $134,2 \pm 2,2 p<0,05$ & $\begin{array}{l}130,2 \pm 2,3 \\
p>0,05 p_{1}>0,05\end{array}$ \\
\hline $\mathrm{KCO}, \mathrm{CM}^{3}$ & $45,2 \pm 2,0$ & $48,2 \pm 2,0 p>0,05$ & $\begin{array}{l}47,6 \pm 1,8 \\
p>0,05 p_{1}>0,05\end{array}$ \\
\hline УО, мл & $84,4 \pm 2,8$ & $84,6 \pm 2,6 p>0,05$ & $\begin{array}{l}81,6 \pm 2,6 \\
p>0,05 p_{1}>0,05\end{array}$ \\
\hline$\Phi \mathrm{B}, \%$ & $64,2 \pm 1,8$ & $64,2 \pm 0,8 p>0,05$ & $\begin{array}{l}63,2 \pm 1,4 \\
p>0,05 p_{1}>0,05\end{array}$ \\
\hline FS\% & $36,2 \pm 0,2$ & $\begin{array}{l}35,0 \pm 0,4 \\
p>0,05\end{array}$ & $\begin{array}{l}34,6 \pm 0,5 \\
p>0,05 p_{1}>0,05\end{array}$ \\
\hline MOC, л & $5,4 \pm 0,4$ & $\begin{array}{l}7,6 \pm 0,2 \\
p<0,01\end{array}$ & $\begin{array}{l}5,8 \pm 0,2 \\
p>0,05 p<0,01\end{array}$ \\
\hline ЧСС, уд/мин & $62,8 \pm 2,8$ & $86,6 \pm 1,8 p<0,01$ & $70,0 \pm 1,4 p>0,05 p_{1}<0,01$ \\
\hline $\begin{array}{l}\text { АД/ср } \\
\text { мм рт.ст. }\end{array}$ & $97,4 \pm 1,2$ & $\begin{array}{l}97,0 \pm 1,6 \\
p>0,05\end{array}$ & $97,1 \pm 1,6 p>0,05 p_{1}<0,05$ \\
\hline СИ л/мин х м² & $3,2 \pm 0,1$ & $3,9 \pm 0,1 p<0,05$ & $3,4 \pm 0,1 \mathrm{p}<0,05 \mathrm{p}_{1}<0,05$ \\
\hline $\begin{array}{l}\text { ОПСС } \\
\text { дин с.сек }\end{array}$ & $\begin{array}{l}1275,3 \pm 38,5 \\
p<0,01\end{array}$ & $\begin{array}{l}1290,2 \pm 90,8 \\
p>0,05 p_{1}<0,05\end{array}$ & $\begin{array}{l}1348,6 \pm 61,1 \\
p>0,05 p_{1}<0,01 p_{2}>0,05\end{array}$ \\
\hline
\end{tabular}

Примечание: различия с показателями - p-контрольной группы, р1 - до операции

но. У этих больных также отмечали увеличение сердечного индекса (СИ) - $\mathrm{p}<0,01)$. При прогрессировании степени декомпенсации ОТКН обнаружили тенденцию к уменьшению МОС и СИ, что проявлялось ухудшением сердечной деятельности.

При снижении MOC у больных с суб- и декомпенсированной ОТКН, выявляли достоверное повышение ОПСС до 1290,2 $\pm 90,8$ (р>0,05) и 1398,6 $\pm 61,1$ дин.С.см-5 ( $>>0,05)$, соответственно, по сравнению с показателями ОПСС $1035,3 \pm 38,5$ дин.С.см-5 ( $p<0,01)$.больных с компенсированной ОТКН.

Наблюдения демонстрируют, что в суб- и декомпенсированной стадии ОТКН, и развивающаяся интраабдоминальная гипертензия со сдавлением нижней полой вены, приводит к значительному снижению венозного возврата и прогрессивному снижению сердечного выброса.

При формулировке названий использовалось традиционное деление на нормокинетический, гипокинетический и гиперкинетический типы кровообращения по величине сердечного индекса [1, с. 176; 2, с. 127].

Для компенсированной и субкомпенсированной стадии ОТКН был характерен гипердинамический тип кровообращения с увеличением МОС, причем, у больных с субкомпенсированной ДКН уже наступали нарушения сократительной способности миокарда. Уменьшение УО при декомпенсированной стадии ОТКН, даже при выраженной тахикардии, приводило к уменьшению МОС (гиподинамический тип кровообращения) и значительному снижению сократительной активно- 
Таблица 3. Данные об изменении центральной гемодинамики у больных с субкомпенсированной динамической кишечной непроходимостью после декомпрессии

\begin{tabular}{|c|c|c|c|c|}
\hline Показатель & Контроль & До стентирования & 3 сутки после стентирования & 7 сутки после стентирования \\
\hline КДР, мм & $\begin{array}{l}52,2 \pm 0,6 \\
p>0,05\end{array}$ & $\begin{array}{l}51,0 \pm 0,6 \\
p>0,05\end{array}$ & $\begin{array}{l}51,2 \pm 0,4 \\
p>0,05 p_{1}<0,01\end{array}$ & $\begin{array}{l}51,3 \pm 0,4 \\
p>0,05 p_{1}>0,05\end{array}$ \\
\hline KCP, MM & $32,2 \pm 0,8$ & $\begin{array}{l}36,6 \pm 0,6 \\
p<0,01\end{array}$ & $\begin{array}{l}35,2 \pm 0,5 \\
p<0,05 p_{1}<0,01\end{array}$ & $\begin{array}{l}34,0 \pm 0,5 \\
p>0,05 p_{1}<0,01\end{array}$ \\
\hline КДО, $\mathrm{cm}^{3}$ & $128,8 \pm 3,8$ & $\begin{array}{l}124,2 \pm 3,4 \\
p>0,05\end{array}$ & $\begin{array}{l}125,0 \pm 3,3 \\
p>0,05 p_{1}<0,05\end{array}$ & $\begin{array}{l}125,4 \pm 3,4 \\
p>0,05 p_{1}>0,05\end{array}$ \\
\hline $\mathrm{KCO}, \mathrm{CM}^{3}$ & $45,2 \pm 2,0$ & $\begin{array}{l}57,2 \pm 1,2 \\
p<0,01\end{array}$ & $\begin{array}{l}51,4 \pm 1,4 \\
p<0,05 p_{1}<0,01\end{array}$ & $\begin{array}{l}47,8 \pm 1,2 \\
p>0,05 p_{1}<0,01\end{array}$ \\
\hline УО, мл & $84,4 \pm 2,8$ & $\begin{array}{l}66,8 \pm 3,4 \\
p<0,01\end{array}$ & $\begin{array}{l}73,8 \pm 3,4 \\
p>0,05 p_{1}<0,01\end{array}$ & $\begin{array}{l}78,0 \pm 3,4 \\
p>0,05 p_{1}<0,01\end{array}$ \\
\hline$\Phi \mathrm{B}, \%$ & $64,2 \pm 1,8$ & $\begin{array}{l}53,6 \pm 2,2 \\
p<0,01\end{array}$ & $\begin{array}{l}58,6 \pm 2,2 \\
p<0,05 p_{1}<0,01\end{array}$ & $61,9 \pm 1,6 p>0,05 p_{1}<0,01$ \\
\hline FS\% & $36,2 \pm 0,2$ & $\begin{array}{l}27,8 \pm 2,2 \\
p<0,01\end{array}$ & $\begin{array}{l}31,4 \pm 1,2 \\
p<0,05 p_{1}<0,01\end{array}$ & $\begin{array}{l}33,4 \pm 1,2 \\
p>0,05 p_{1}<0,01\end{array}$ \\
\hline MOC, л & $5,4 \pm 0,4$ & $\begin{array}{l}6,2 \pm 0,2 \\
p>0,05\end{array}$ & $\begin{array}{l}6,1 \pm 0,2 \\
p>0,05 p_{1}>0,05\end{array}$ & $\begin{array}{l}5,9 \pm 0,4 \\
p>0,05 p_{1}>0,05\end{array}$ \\
\hline ЧСС, уд/мин & $62,8 \pm 2,8$ & $\begin{array}{l}92,4 \pm 2,2 \\
p<0,01\end{array}$ & $\begin{array}{l}83,4 \pm 1,6 \\
p<0,05 p_{1}<0,01\end{array}$ & $\begin{array}{l}78,0 \pm 1,4 \\
p<0,05 p_{1}<0,01\end{array}$ \\
\hline АД/ср, мм рт.ст. & $97,4 \pm 1,2$ & $\begin{array}{l}96,8 \pm 1,2 \\
p>0,05\end{array}$ & $\begin{array}{l}97,0 \pm 1,2 \\
p>0,05 p_{1}>0,05\end{array}$ & $\begin{array}{l}97,0 \pm 1,2 \\
p>0,05 p_{1}>0,05\end{array}$ \\
\hline СИл/мин $\times \mathrm{M}^{2}$ & $3,2 \pm 0,1$ & $3,2 \pm 0,2 p<0,05$ & $3,3 \pm 0,1 p<0,05 p_{1}>0,05$ & $3,4 \pm 0,1 p<0,05 p_{1}>0,05$ \\
\hline $\begin{array}{l}\text { ОПСС } \\
\text { дин с.сек }{ }^{-5}\end{array}$ & $1424,4 \pm 48,7$ & $\begin{array}{l}1258,3 \pm 38,5 \\
p<0,01\end{array}$ & $\begin{array}{l}1268,2 \pm 60,4 \\
p>0,05 p_{l}<0,05\end{array}$ & $\begin{array}{l}1484,6 \pm 61,1 \\
p>0,05 p_{1}<0,01 p_{2}>0,05\end{array}$ \\
\hline
\end{tabular}

Примечание: различия с показателями - p-контрольной группы, р1 - до операции

сти миокарда. При гипокинетическом типе основными корректирующими препаратами являлись лекарственные средства из группы сердечных гликозидов.

У больных с компенсированной ОТКН, когда выявляли гипердинамический тип кровообращения, восстановление показателей гемодинамики отметили на 3 сутки после декомпрессии (табл. 2). К этому сроку от-

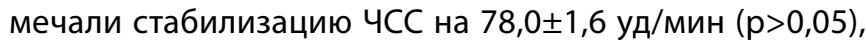

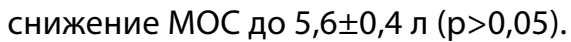

При суб- и декомпенсированной ОТКН, когда определяли нарушения сократительной активности сердечной мышцы со снижением УО и МОС, восстановление гемодинамических показателей отмечали в более поздние сроки.

После транстуморальной декомпрессии у больных с субкомпенсированной ОТКН (табл. 3) на 3 сутки после операции выявили тенденцию к снижению чСС и увеличению УО, при этом динамики показателей МОС не было. Нормализация гемодинамических показателей у этих больных наступала лишь на 7 сутки, когда

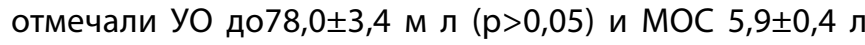
$(\mathrm{p}>0,05)$.

При анализе результатов исследования больных, стентированных по поводу декомпенсировнной ОТКН - (табл. 3), на 3 и 7 сутки после операции отмечали прогрессирующее улучшение показателей центральной гемодинамики со снижением чСС, увеличением УО и ФВ.

Однако нормальных значений гемодинамические показатели достигали только к 11-12 суткам, когда отмечали снижение чСС до 78,6 $\pm 1,4$ мм рт.ст.(p>0,05, уве-

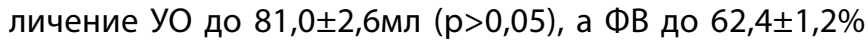
( $p>0,05$, стабилизации MOC на 5,5 $\pm 0,2$ л ( $p>0,05)$. 
Таблица 4. Данные об изменении центральной гемодинамики у больных с декомпенсированной ОТКН после стентирования кишки

\begin{tabular}{|c|c|c|c|c|c|}
\hline Показатель & Контроль & До стентирования & $\begin{array}{l}3 \text { сутки после } \\
\text { стентирования }\end{array}$ & $\begin{array}{l}7 \text { сутки } \\
\text { после } \\
\text { стентирования }\end{array}$ & $\begin{array}{l}11 \text { сутки после } \\
\text { стентирования }\end{array}$ \\
\hline КДР, мм & $\begin{array}{l}52,2 \pm 0,6 \\
p>0,05\end{array}$ & $\begin{array}{l}51,2 \pm 0,6 \\
p>0,05\end{array}$ & $\begin{array}{l}50,9 \pm 0,6 \\
p>0,05 p_{1}>0,05\end{array}$ & $\begin{array}{l}51,2 \pm 0,6 p>0,05 \\
p_{1}>0,05\end{array}$ & $\begin{array}{l}51,3 \pm 0,6 p>0,05 \\
p_{1}>0,05\end{array}$ \\
\hline KCP, MM & $32,2 \pm 0,8$ & $\begin{array}{l}39,4 \pm 0,4 \\
p<0,01\end{array}$ & $\begin{array}{l}35,8 \pm 0,4 \\
p<0,01 p_{1}<0,01\end{array}$ & $\begin{array}{l}34,6 \pm 0,4 \\
p>0,05 p_{1}<0,01\end{array}$ & $\begin{array}{l}32,9 \pm 0,4 \\
p>0,05 p_{1}<0,01\end{array}$ \\
\hline КДО, см ${ }^{3}$ & $128,8 \pm 3,8$ & $\begin{array}{l}125,6 \pm 3,1 \\
p>0,05\end{array}$ & $\begin{array}{l}123,6 \pm 3,1 \\
p>0,05 p_{1}>0,05\end{array}$ & $\begin{array}{l}124,6 \pm 3,1 \\
p>0,05 p_{1}>0,05\end{array}$ & $\begin{array}{l}125,3 \pm 2,6 \\
p>0,05 p_{1}>0,05\end{array}$ \\
\hline $\mathrm{KCO}, \mathrm{CM}^{3}$ & $45,2 \pm 2,0$ & $\begin{array}{l}66,8 \pm 1,8 \\
p<0,01\end{array}$ & $\begin{array}{l}53,8 \pm 1,8 \\
p<0,01 p_{1}<0,01\end{array}$ & $\begin{array}{l}49,8 \pm 1,2 \\
p>0,05 p_{1}<0,01\end{array}$ & $\begin{array}{l}44,2 \pm 1,0 \\
p>0,05 p_{1}<0,01\end{array}$ \\
\hline УО, мл & $84,4 \pm 2,8$ & $\begin{array}{l}58,6 \pm 2 \\
p<0,01\end{array}$ & $\begin{array}{l}68,6 \pm 2,9 \\
p<0,01 p_{1}<0,05\end{array}$ & $\begin{array}{l}75,6 \pm 2,2 \\
p<0,05 p_{1}<0,01\end{array}$ & $\begin{array}{l}81,0 \pm 2,6 \\
p>0,05 p_{1}<0,01\end{array}$ \\
\hline$\Phi \mathrm{B}, \%$ & $64,2 \pm 1,8$ & $\begin{array}{l}46,4 \pm 2,4 \\
p<0,01\end{array}$ & $\begin{array}{l}56,4 \pm 1,4 \\
p<0,01 p_{1}<0,01\end{array}$ & $\begin{array}{l}60,4 \pm 0,4 \\
p<0,05 p_{1}<0,01\end{array}$ & $\begin{array}{l}62,4 \pm 1,2 \\
p>0,05 p_{1}<0,01\end{array}$ \\
\hline FS\% & $36,2 \pm 0,2$ & $\begin{array}{l}23,8 \pm 1,4 \\
p<0,01\end{array}$ & $\begin{array}{l}29,8 \pm 1,2 \\
p<0,01 p_{1}<0,01\end{array}$ & $\begin{array}{l}32,4 \pm 1,0 \\
p<0,05 p_{1}<0,01\end{array}$ & $\begin{array}{l}35,5 \pm 1,0 \\
p>0,05 p_{1}<0,01\end{array}$ \\
\hline MOC, л & $5,4 \pm 0,4$ & $\begin{array}{l}5,7 \pm 0,2 \\
p>0,05\end{array}$ & $\begin{array}{l}5,7 \pm 0,2 \\
p>0,05 p_{1}>0,05\end{array}$ & $\begin{array}{l}5,5 \pm 0,2 \\
p>0,05 p_{1}>0,05\end{array}$ & $\begin{array}{l}5,5 \pm 0,2 \\
p>0,05 p_{1}>0,05\end{array}$ \\
\hline ЧСС, уд/мин & $62,8 \pm 2,8$ & $\begin{array}{l}99,8 \pm 2,2 \\
p<0,01\end{array}$ & $\begin{array}{l}84,8 \pm 1,2 \\
p<0,01 p_{1}<0,01\end{array}$ & $\begin{array}{l}79,2 \pm 1,2 \\
p<0,01 p_{1}<0,01\end{array}$ & $\begin{array}{l}78,6 \pm 1,4 \\
p>0,05 p_{1}<0,01\end{array}$ \\
\hline АД/ср, мм рт.ст. & $97,4 \pm 1,2$ & $\begin{array}{l}96,8 \pm 1,3 \\
p>0,05\end{array}$ & $\begin{array}{l}96,2 \pm 1,3 \\
p>0,05 p_{1}>0,05\end{array}$ & $\begin{array}{l}96,4 \pm 1,3 \\
p>0,05 p_{1}>0,01\end{array}$ & $98,2 \pm 0,8 p>0,05 p_{1}>0,05$ \\
\hline $\begin{array}{l}\text { СИ } \\
\text { л/мин } \times \mathrm{M}^{2}\end{array}$ & $3,2 \pm 0,1$ & $\begin{array}{l}2,9 \pm 0,4 \\
p>0,05\end{array}$ & $\begin{array}{l}3,3 \pm 0,4 \\
p>0,05 p_{1}>0,05\end{array}$ & $\begin{array}{l}3,3 \pm 0,4 \\
p>0,05 p_{1}>0,05\end{array}$ & $\begin{array}{l}3,4 \pm 0,2 \\
p>0,05 p_{1}>0,05\end{array}$ \\
\hline $\begin{array}{l}\text { ОПСС } \\
\text { дин с.сек-5 }\end{array}$ & $1426,4 \pm 44,5$ & $1492,4 \pm 66,8$ & $1462,2 \pm 46,4$ & $1442,2 \pm 26,4$ & $\begin{array}{l}1422,4 \pm 26,2 \\
p>0,05 p_{1}<0,01 p_{2}>0,05\end{array}$ \\
\hline
\end{tabular}

Примечание: различия с показателями — p-контрольной группы, р1-до операции

При уменьшении внутрибрюшной гипертензии СИ достоверно увеличивался: с 2,9 $20,4 л$ х мин/м (1-й день) до 3,4 л/мин х м2 (11 -й день).

Наиболее часто встречающимся гемодинамическим осложнением являлось нарушение сердечного ритма, чаще фибрилляция предсердий или суправентрикулярная тахикардия. После применения индивидуализированной схемы коррекции у больных сердечно-сосудистые осложнения были отмечены только дважды.
Таким образом, исследования показали, что эндоскопическая декомпрессия (транстуморальное стентирование) при ОТКН способствуют уменьшению интраабдоминальной гипертензии и позволяет у всех пациентов с компенсированной и субкомпенсированной кишечной непроходимостью восстановить гемодинамические показатели в течение 7-8 суток, а у наиболее тяжелых больных с декомпенсированной кишечной непроходимостью приблизить сроки нормализации только к 10-12суткам после декомпрессии. 


\section{ЛИТЕРАТУРА}

1. Лебедева Р.Н. Осложнения в системе кровообращения после хирургических вмешательств. М.: «Медицина» 1979.

2. Шхвацабая И.К., Константинов Е.Н., Гундаров И.А. 0 новом подходе к пониманию гемодинамической нормы // Кардиология. 1981. № 3.

3. Чадаев А.П., Хрипун А.И. Перитонит и внутрибрюшное давление. М. 2003.

4. Bloomfield G.L., Ridings P.C., Blocher C.R. et al. Effects of increased intra-abdominal pressure upon intracranial and cerebral perfusion pressure before and after volume expansion // J Trauma. 1996. Vol. 6.

5. Boone B., Zureikat A., Hughes S. et al. Abdominal compartment syndrome is an early, lethal complication of acute pancreatitis. Am. Surg. 2013.

6. Gargiulo N.J., Simon R.J., Leon W., Machiedo G.W. Hemorrhage exacerbates bacterial translocation at low levels of intra-abdominal pressure // Arch. Surg. 1998. Vol. 133.

7. Chevallier P., Baque P., Benchimol D. Treatment of colorectal obstruction with self-expanding metallic stents under fluoroscopic guidance. J Radiol 2002.

8. Cheatham M.L., White M.W., Sagraves S.G., Johnson J.L., Block E.F. Abdominal perfusion pressure: a superior parameter in the assessment of intra-abdominal hypertension // J. Trauma. 2000 0ct. 49(4); discussion 626-7.

9. Cortes-Puentes G., Gard K., Adams A. et al. Value and limitations of transpulmonary pressure calculations during intraabdominal hypertension. Crit. Care Med. 2013; 41 (8).

10. Malbrain M.L.N.G. Intra-abdominal pressure in the ICU: Clinical tool or toy? // Yearbook of Intens. Care and Emerg. Med. / Vincent JL (ed). — Berlin: SpringerVerlag. 2001.

11. Malbrain, M.L.N.G. Resultsfirom the international conference of experts on intra-abdominal hypertension and abdominal compartment syndrome. Part 1. / M.L.N.G.

12. Malbrain, M.L. Cheatham, A. Kirkpatrick // Intensive Care Med. 2006. № 32.

13. Savino J.A., Cerabona T., Agarwal N., Byrne D. Manipulation of ascitic fluid pressure in cirrhotics to optimize hemodynamic and renal function // Ann. Surg. 1988. Vol. 208, № 4.

14. Sugrue M., Jones F., Lee A. et al. Intra-abdominal pressure and gastric intramucosal pH: Is there an association? // World J. Surg. 1996. Vol. 20.

15. Sugrue M., Jones F., Janjua K.J. et al. Temporary abdominal closure: a prospective evaluation of its effects on renal and respiratory function // J. Trauma. 1998. Vol. 45.

16. Sukhotnik I., Mogilner J., Hayari L. et al. Effect of elevated in- tra-abdominal pressure and $100 \%$ oxygen on superior mesenteric artery blood flow and enterocyte turnover in a rat. Pediat. Surg. Int. 2008; 24 (12).

17. Pickhardt P.J., Shimony J.S., Heiken J.P. et al. The abdominal compartment syndrome: CT findings // Amer. J. Rent. 1999. Vol. 173.

18. Richardson D., Wasserman A., Patterson J. General and regional circulatory responses to change in blood pH and carbon dioxide tension // J. Clin. Invest. 1961. Vol. 40.

19. Wachsberg R.H., Sebastiano L.L., Levine C.D. Narrowing of the upper abdominal inferior vena cava in patients with elevated intraabdominal pressure // Abdom. Imaging. 1998. Vol. 23, № 1.

( ) Багдасаров Валерий Вартанович, Багдасарова Елена Анатольевна, Елеев Алим Анатольевич ( Alimeleev@gmail.com ).

Журнал «Современная наука: актуальные проблемы теории и практики» 